\title{
Diagnostic Criteria of Postoperative Cognitive Dysfunction: A Focused Systematic Review
}

\author{
Kim van Sinderen, Lothar A. Schwarte 1 , and Patrick Schober $(\mathbb{D}$ \\ Department of Anesthesiology, Amsterdam University Medical Centers, Vrije Universiteit Amsterdam, Amsterdam, Netherlands \\ Correspondence should be addressed to Patrick Schober; p.schober@vumc.nl
}

Received 21 May 2020; Revised 24 September 2020; Accepted 2 November 2020; Published 17 November 2020

Academic Editor: Yukio Hayashi

Copyright (c) $2020 \mathrm{Kim}$ van Sinderen et al. This is an open access article distributed under the Creative Commons Attribution License, which permits unrestricted use, distribution, and reproduction in any medium, provided the original work is properly cited.

\begin{abstract}
Postoperative Cognitive Dysfunction (POCD) is characterized by a deterioration in cognitive performance after surgery and is increasingly addressed in research studies. However, a uniform definition of POCD seems to be lacking, which is a major threat to clinical research in this area. We performed a focused systematic review to determine the current degree of heterogeneity in how POCD is defined across studies and to identify those diagnostic criteria that are used most commonly. The search identified 173 records, of which 30 were included. Neurocognitive testing was most commonly performed shortly before surgery and at 7 days postoperatively. A variety of neurocognitive tests were used to test a range of cognitive domains, including complex attention, language, executive functioning, perceptual-motor function, and learning and memory. The tests that were used most commonly were the Mini-Mental State Examination, the digit span test, the trail making test part A, and the digit symbol substitution test, but consensus on which test result would be considered "positive" for POCD was sparse. The results of this systematic review suggest the lack of a consistent approach towards defining POCD. However, commonalities were identified which may serve as a common denominator for deriving consensus-based diagnostic guidelines for POCD.
\end{abstract}

\section{Introduction}

Postoperative Cognitive Dysfunction (POCD) is characterized by a deterioration in cognitive performance after surgery and is particularly prevalent in elderly patients [1]. The cognitive decline is usually transient, but can be a substantial threat to the quality of life as it occasionally persists for months to years after surgery. The pathophysiology and risk factors are incompletely understood, and effective strategies for prevention and treatment yet need to be established. Thus, POCD is the target of an increasing amount of research studies and reviews published in leading anesthesia journals [2-4].

Despite the clinical relevance and the need for standardized research, a uniform definition of POCD seems to be lacking. A systematic review in 2010 revealed a major inconsistency in diagnostic criteria, neurocognitive tests, and timing of the assessment of POCD across studies in cardiac surgery [5]. The need for uniform terminology and diagnostic criteria in POCD research has in the meantime been widely recognized, and recommendations on uniform nomenclature for perioperative neurocognitive disorders have been published [6]. Yet, it is unclear whether POCD research has recently adopted a more uniform approach to defining POCD and which criteria and tests are currently most commonly used to define POCD.

We thus performed a focused systematic review, limited to literature published in the last two years, to determine the current degree of heterogeneity in how POCD is defined and diagnosed across research studies. The results of this review may identify those tests and criteria that are currently used most often and can serve as a common denominator to derive a broader consensus on which tests and criteria should actually be used to diagnose POCD.

\section{Methods}

PubMed was searched for original research studies on POCD in adult patients published between January 1, 2018 and January 14, 2020, using the following search strategy: 


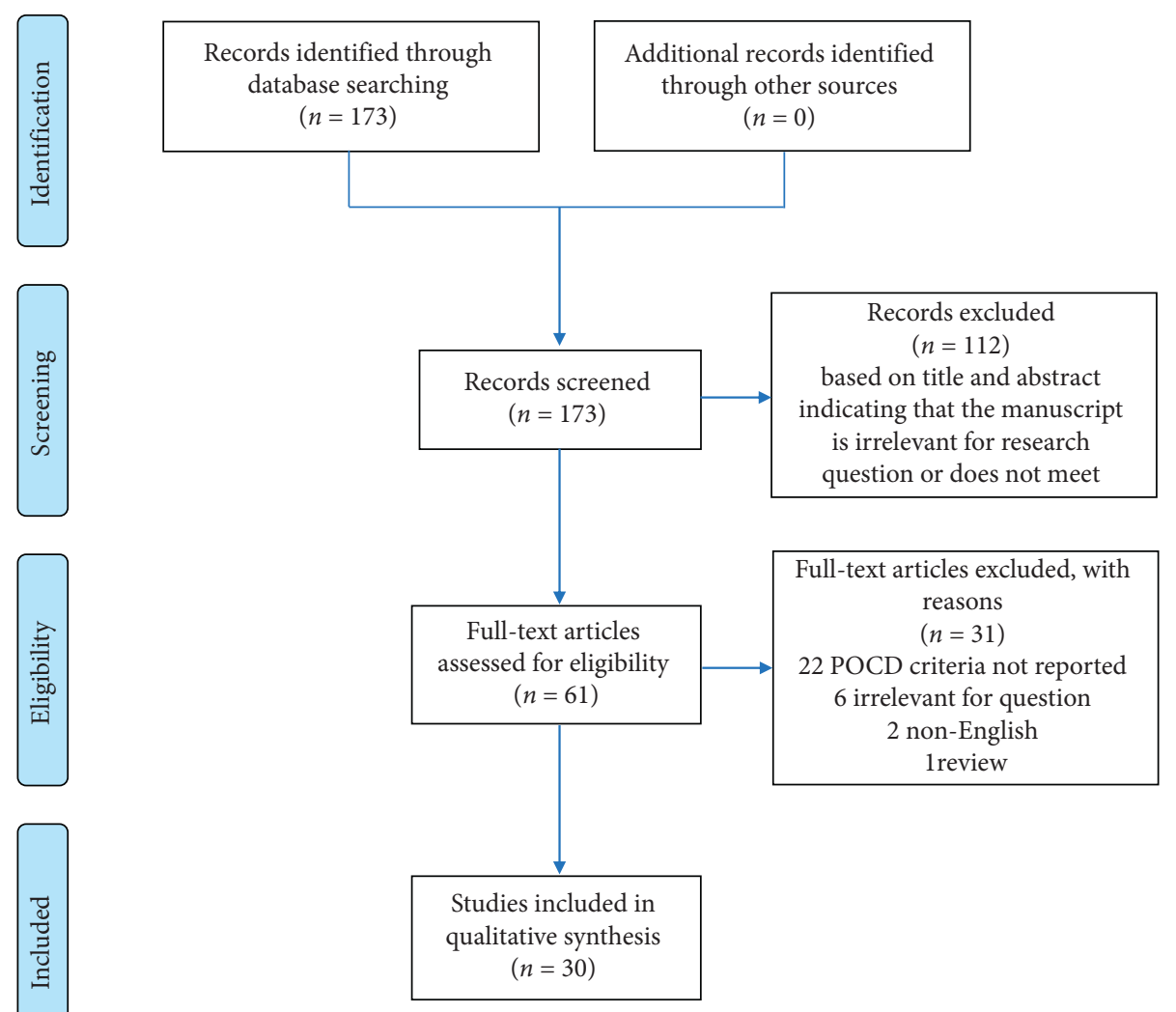

FIgURE 1: Flow diagram of studies included in the systematic review.

("Postoperative Cognitive Complications" [Mesh] OR "Postoperative Period" [Mesh] OR Postoperative [tiab] OR Post-operative [tiab]) AND ("Cognitive Dysfunction" [Mesh] OR (cognitive dysfunction [tiab] OR cognitive dysfunctioning [tiab] OR cognitive dysfunctions [tiab]) OR cognitive decline [tiab]) AND (("2018/01/01" [PDAT]: “2020/01/14” [PDAT]) AND “adult” [MeSH Terms]). Titles and abstracts were screened by 2 authors, and the full text of potentially relevant articles was retrieved. Original research studies on human subjects, published in English language, that explicitly reported which diagnostic criteria had been used to define POCD, were eligible for inclusion. Data on study and patient characteristics, POCD criteria, and tests used to assess POCD, as well as the time points at which the tests were performed, were extracted using a standardized data extraction form.

Because the research question does not lend itself to quantitative pooling of data across studies, and due to substantial heterogeneity across studies, a meta-analysis of the collected data was not performed, and all results are presented descriptively.

\section{Results}

The PubMed search identified 173 records, and 61 full-text articles were assessed for eligibility (Figure 1). Of these, 30 studies reporting POCD criteria were included in this systematic review [7-36]. Seven studies report data of a randomized controlled trial, while the rest were observational study designs (cohort, case-control, and longitudinal studies). The study characteristics, POCD criteria, and POCD assessment tests and time points are summarized in Table 1.

In the majority of studies $(n=25)$, baseline neurocognitive performance was measured either on the day of surgery or on the day before surgery, while the other studies used varying preoperative time intervals. The most frequently used follow-up time was 7 days after surgery $(n=23)$. Ten out of the 30 studies followed patients up over a longer period of time, up to one year after surgery.

A variety of different neurocognitive tests (Table 2) were used to test a range of cognitive domains, including complex attention, language, executive functioning, perceptual-motor function, and learning and memory. We refer to Sachdev et al. [37] for a detailed description of the different cognitive domains and to Evered and Silbert [3] for an overview over which domains are being tested by which neurocognitive test. Nine studies included in our systematic review relied on only one test, either the Mini-Mental State Examination (MMSE, $n=5$ ) or the Montreal Cognitive Assessment (MoCA, $n=4$ ), both of which actually test several neurocognitive domains. The other studies used a battery of different tests for different domains, and some of these studies also additionally included the MMSE $(n=9)$ or MoCA $(n=2)$ tests. Besides the MMSE, the most commonly used tests were the digit span test (forward test $n=13$, 
TABLE 1: Characteristics of studies reporting diagnostic criteria to define POCD.

\begin{tabular}{|c|c|c|c|c|c|c|}
\hline Author (year) & Study type/topic & Study population(s) & Type of surgery & POCD criteria & $\begin{array}{c}\text { POCD } \\
\text { assessment }\end{array}$ & $\begin{array}{c}\text { Timepoint of } \\
\text { assessment }\end{array}$ \\
\hline $\begin{array}{l}\text { Claes et al., } \\
2018 \text { [7] }\end{array}$ & $\begin{array}{c}\text { Prospective cohort } \\
\text { study } \\
\text { Incidence POCD } \\
\text { cochlear } \\
\text { implantation }\end{array}$ & $\begin{array}{c}26 \text { (16 male, } 10 \\
\text { female) } \\
\geq 55 \text { years } \\
\text { POCD incidence } 7 \\
\text { days post-op: } 11.5 \%\end{array}$ & $\begin{array}{c}\text { Cochlear } \\
\text { implantation }\end{array}$ & $\begin{array}{c}\text { A } z \text {-score of change } \\
\text { in MoCA scores } \\
\geq 1.96 .\end{array}$ & (i) $\mathrm{MoCA}$ & $\begin{array}{l}\text { (i) Baseline: } \\
\text { (ii) Day of } \\
\text { surgery } \\
\text { (iii) Post-op: } \\
\text { (iv) } 7 \text { days }\end{array}$ \\
\hline $\begin{array}{l}\text { Duan et al., } \\
2018[8]\end{array}$ & $\begin{array}{l}\text { Prospective } \\
\text { observational study } \\
\text { Glial cell line } \\
\text { derived neurotropic } \\
\text { factor as predictor } \\
\text { for POCD }\end{array}$ & $\begin{array}{c}80 \text { (36 male/44 } \\
\text { female) } \\
20 \text { healthy controls } \\
>50 \text { years } \\
\text { POCD incidence } 7 \\
\text { days post-op: } 47.5 \%\end{array}$ & $\begin{array}{c}\text { Elective heart } \\
\text { valve replacement }\end{array}$ & $\begin{array}{l}\text { At least } 2 z \text { scores } \\
\quad>1.96 \text {. } \\
\text { Compared to } 20 \\
\text { healthy controls } \\
\text { matched by gender, } \\
\text { age, and education. }\end{array}$ & $\begin{array}{l}\text { (i) MMSE } \\
\text { (ii) Digit span test } \\
\text { (iii) Trail making } \\
\text { test A } \\
\text { (iv) Digit symbol } \\
\text { (v) Modalities } \\
\text { clock drawing test }\end{array}$ & $\begin{array}{l}\text { (i) Baseline: } \\
\text { (ii) } 1 \text { day } \\
\text { before } \\
\text { surgery } \\
\text { (iii) Post-op: } \\
\text { (iv) } 7 \text { days }\end{array}$ \\
\hline $\begin{array}{l}\text { Gong et al., } \\
2018 \text { [9] }\end{array}$ & $\begin{array}{l}\text { Cohort study } \\
\text { Incidence of POCD } \\
\text { laparoscopic vs. } \\
\text { open surgery }\end{array}$ & $\begin{array}{c}70 \text { (41 male/29 } \\
\text { female) } \\
\text { 32-85 years } \\
\text { POCD incidence } 7 \\
\text { days post-op: } \\
\text { Open: } 18.7 \% \\
\text { Laparoscopic: } 12.5 \%\end{array}$ & $\begin{array}{l}\text { Abdominal } \\
\text { surgery }\end{array}$ & $\begin{array}{l}\text { Patients with an } \\
\text { MMSE score } \\
\text { reduction of two. }\end{array}$ & (i) MMSE & $\begin{array}{l}\text { (i) Baseline: } \\
\text { (ii) } 1 \text { day } \\
\text { before } \\
\text { (iii) Post-op: } \\
\text { (iv) } 1 \text { and } 7 \\
\text { days }\end{array}$ \\
\hline $\begin{array}{l}\text { Hayashi et al., } \\
2018 \text { [10] }\end{array}$ & $\begin{array}{l}\text { Secondary analysis } \\
\text { 6-minute walk } \\
\text { distance and POCD }\end{array}$ & $\begin{array}{c}181 \text { (121 male/60 } \\
\text { female) } \\
\geq 60 \text { years } \\
\text { POCD incidence } 14 \\
\text { days post-op: } 28 \%\end{array}$ & Cardiac surgery & $\begin{array}{l}\text { Significant decline in } \\
\text { cognitive function } \\
\text { based on a decrease } \\
\text { of } 2 \text { or more in the } \\
\text { MMSE score. }\end{array}$ & (i) MMSE & $\begin{array}{l}\text { (i) Baseline: } \\
\text { (ii) } \\
\text { Admission } \\
\text { (iii) Post-op: } \\
\text { (iv) } 14 \text { days }\end{array}$ \\
\hline $\begin{array}{l}\text { Hou et al., } \\
2018[11]\end{array}$ & $\begin{array}{c}\text { RCT } \\
\text { Deep versus light } \\
\text { anesthesia and } \\
\text { POCD }\end{array}$ & $\begin{array}{c}60 \text { (26 male/34 } \\
\text { female) } \\
\geq 60 \text { years } \\
\text { POCD incidence } 1 \\
\text { day post-op: } \\
\text { Low doses: } 20 \% \\
\text { High doses: } 3 \%\end{array}$ & $\begin{array}{l}\text { Total knee } \\
\text { replacement }\end{array}$ & $\begin{array}{c}A z \text {-score of }>1.96 \\
\text { using cross- } \\
\text { reference. }\end{array}$ & (i) $\mathrm{MoCA}$ & $\begin{array}{l}\text { (i) Baseline: } \\
\text { (ii) Day of } \\
\text { surgery } \\
\text { (iii) Post-op: } \\
\text { (iv) 1, 3, and } 7 \\
\text { days }\end{array}$ \\
\hline $\begin{array}{l}\text { Konishi et al., } \\
2018 \text { [12] }\end{array}$ & $\begin{array}{l}\text { Secondary analysis } \\
\text { of prospective } \\
\text { observational study } \\
\text { (ACE study) } \\
\text { Incidence of POCD } \\
\text { sevoflurane vs. } \\
\text { propofol }\end{array}$ & $\begin{array}{c}121 \text { sevoflurane (77 } \\
\text { male/44 female), } 171 \\
\text { propofol (114 male/ } \\
57 \text { female) } \\
\geq 60 \text { years } \\
\text { POCD incidence } 7 \\
\text { days post-op: } \\
\text { Sevoflurane: } 20.2 \% \\
\text { Propofol: } 15 \%\end{array}$ & $\begin{array}{l}\text { Total hip } \\
\text { replacement }\end{array}$ & $\begin{array}{l}\geq 2 \text { tests were at least } \\
1.96 \text { SD lower than } \\
\text { the mean score of the } \\
\text { matched control } \\
\text { group after adjusting } \\
\text { for expected change } \\
\text { over time using } \\
\text { controls or the } \\
\text { combined } z \text {-score } \\
\text { was less than }-1.96 \text {. }\end{array}$ & $\begin{array}{l}\text { (i) Auditory } \\
\text { verbal learning } \\
\text { test } \\
\text { (ii) Trail making } \\
\text { test (parts A and } \\
\text { B) } \\
\text { (iii) Digit symbol } \\
\text { substitution test } \\
\text { (iv) Controlled } \\
\text { oral word } \\
\text { association test } \\
\text { (v) CERAD } \\
\text { semantic fluency } \\
\text { test (animals) } \\
\text { (vi) Grooved } \\
\text { pegboard test } \\
\text { (both hands) }\end{array}$ & $\begin{array}{l}\text { (i) Baseline: } \\
\text { (ii) The week } \\
\text { before } \\
\text { surgery } \\
\text { (iii) Post-op: } \\
\text { (iv) } 7 \text { days } \\
\text { 3,12 months }\end{array}$ \\
\hline
\end{tabular}


TABle 1: Continued.

\begin{tabular}{|c|c|c|c|c|c|c|}
\hline Author (year) & Study type/topic & Study population(s) & Type of surgery & POCD criteria & $\begin{array}{c}\text { POCD } \\
\text { assessment }\end{array}$ & $\begin{array}{c}\text { Timepoint of } \\
\text { assessment }\end{array}$ \\
\hline $\begin{array}{l}\text { Kumpaitiene } \\
\text { et al., } 2018 \\
{[13]}\end{array}$ & $\begin{array}{c}\text { Prospective } \\
\text { observational study } \\
\text { POCD and } \\
\text { decreased cerebral } \\
\text { oxygen saturation }\end{array}$ & $\begin{array}{c}59 \text { (32 male/24 } \\
\text { female) } \\
\geq 60 \text { years } \\
\text { POCD incidence } \\
7-10 \text { days post-op: } \\
37 \%\end{array}$ & $\begin{array}{l}\text { Coronary artery } \\
\text { bypass graft } \\
\text { surgery }\end{array}$ & $\begin{array}{c}\text { The sum of all } z \text { - } \\
\text { scores was }>2 \text { or at } \\
\text { least two } z \text {-scores for } \\
\text { separate tests were } \\
>2 \text {. }\end{array}$ & $\begin{array}{l}\text { (i) MMSE } \\
\text { (ii) Rey auditory } \\
\text { verbal learning } \\
\text { test } \\
\text { (iii) Digit symbol } \\
\text { substitution test } \\
\text { and Schulte table } \\
\text { (iv) Digit span } \\
\text { test } \\
\text { (v) Trail making } \\
\text { test (parts A and } \\
\text { B) }\end{array}$ & $\begin{array}{l}\text { (i) Baseline: } \\
\text { (ii) Day } \\
\text { before } \\
\text { surgery } \\
\text { (iii) Post-op: } \\
\text { (iv) 7-10 days }\end{array}$ \\
\hline $\begin{array}{l}\text { Şahan et al., } \\
2018 \text { [14] }\end{array}$ & $\begin{array}{c}\text { Pilot study } \\
\text { Cerebral oxygen } \\
\text { changes }\end{array}$ & $\begin{array}{c}40 \text { (34 male/6 } \\
\text { female) } \\
\geq 60 \text { years } \\
\text { POCD incidence } 7 \\
\text { days post-op: } \\
45 \% \text { in target group } \\
41 \% \text { in control } \\
\text { group }\end{array}$ & Coronary surgery & $\begin{array}{c}\text { A drop of } 1 \text { standard } \\
\text { deviation from } \\
\text { baseline on two or } \\
\text { more } \\
\text { neuropsychological } \\
\text { tests. }\end{array}$ & $\begin{array}{l}\text { (i) Logical } \\
\text { memory subtest } \\
\text { (ii) Clock drawing } \\
\text { test } \\
\text { Visuospatial skill } \\
\text { test } \\
\text { (iii) Word list } \\
\text { generation test } \\
\text { (iv) Digit span } \\
\text { subtest }\end{array}$ & $\begin{array}{l}\text { (i) Baseline: } \\
\text { (ii) 2-3 days } \\
\text { before } \\
\text { surgery } \\
\text { (iii) Post-op: } \\
7 \text { days } \\
\text { (iv) } 3 \text { months }\end{array}$ \\
\hline $\begin{array}{l}\text { Zhang et al., } \\
2018 \text { [15] }\end{array}$ & $\begin{array}{l}\text { Case control study } \\
\text { Goal-directed fluid } \\
\text { therapy }\end{array}$ & $\begin{array}{c}60 \text { (40 male } / 20 \\
\text { female) } \\
\geq 60 \\
\text { POCD incidence } \\
21.67 \%\end{array}$ & $\begin{array}{l}\text { Spinal stenosis } \\
\text { surgery }\end{array}$ & $\begin{array}{c}\text { If one standard } \\
\text { deviation of MoCA } \\
\text { score was decreased } \\
\text { one or more after } \\
\text { surgery. }\end{array}$ & (i) MoCA & $\begin{array}{l}\text { (i) Baseline: } \\
\text { (ii) } 1 \text { day } \\
\text { before } \\
\text { surgery } \\
\text { (iii) Post-op: } \\
\text { (iv) } 1,3 \text {, and } 7 \\
\text { days }\end{array}$ \\
\hline $\begin{array}{l}\text { Zhang et al., } \\
2018 \text { [16] }\end{array}$ & $\begin{array}{l}\text { Cohort study } \\
\text { Propofol versus } \\
\text { sevoflurane and } \\
\text { cognitive function }\end{array}$ & $\begin{array}{l}379 \text { (263 male/116 } \\
\text { female) } \\
\geq 65 \text { and <90 years } \\
\text { POCD incidence } 7 \\
\text { days post-op: } \\
\text { Sevoflurane: } 23.2 \% \\
\text { propofol: } 19.0 \%\end{array}$ & $\begin{array}{c}\text { Major cancer } \\
\text { surgery ( } \geq 2 \text { hours) }\end{array}$ & $\begin{array}{c}\text { Two } z \text { scores in } \\
\text { individual tests or the } \\
\text { combined } z \text { score } \\
\leq 1.96 .\end{array}$ & $\begin{array}{l}\text { (i) Mental control } \\
\text { (ii) Paired } \\
\text { associate verbal } \\
\text { learning } \\
\text { (iii) Grooved } \\
\text { pegboard (both } \\
\text { hands) } \\
\text { (iv) Visual } \\
\text { retention } \\
\text { (v) Digit symbol } \\
\text { (vi) Trail making } \\
\text { (part A) } \\
\text { (vii) Digit span } \\
\text { (forward and } \\
\text { backward) }\end{array}$ & $\begin{array}{l}\text { (i) Baseline: } \\
\text { (ii) Before } \\
\text { surgery } \\
\text { (iii) Post-op: } \\
\text { (iv) } 7 \text { days }\end{array}$ \\
\hline
\end{tabular}


TABle 1: Continued.

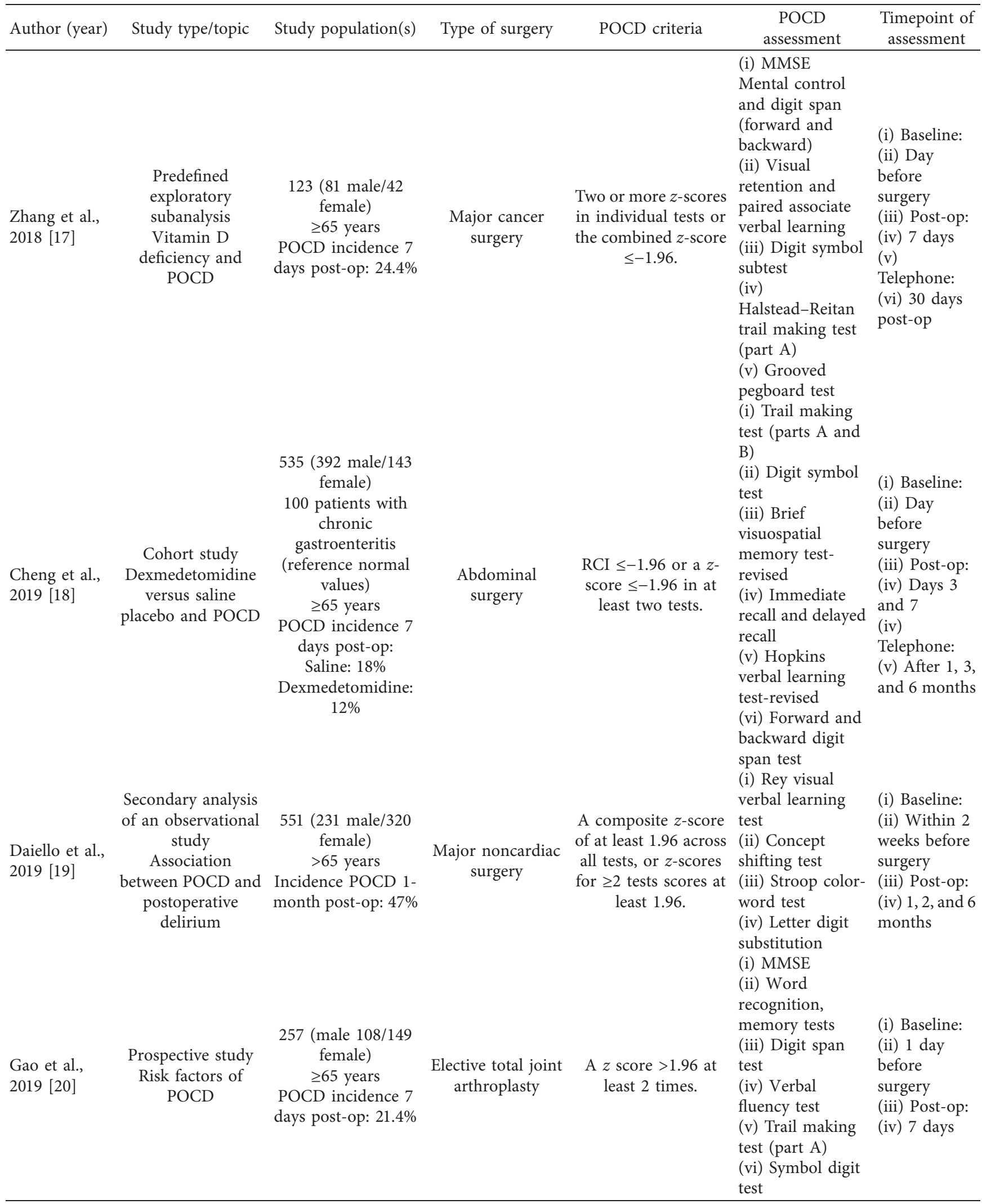


TABle 1: Continued.

\begin{tabular}{|c|c|c|c|c|c|c|}
\hline Author (year) & Study type/topic & Study population(s) & Type of surgery & POCD criteria & $\begin{array}{c}\text { POCD } \\
\text { assessment }\end{array}$ & $\begin{array}{c}\text { Timepoint of } \\
\text { assessment }\end{array}$ \\
\hline $\begin{array}{l}\text { Han et al., } \\
2019 \text { [21] }\end{array}$ & $\begin{array}{l}\text { Cohort study } \\
\text { Cortisol ratio in } \\
\text { saliva as predictor } \\
\text { for POCD }\end{array}$ & $\begin{array}{c}94 \text { (65 male/29 } \\
\text { female) } \\
\text { >60 years } \\
\text { POCD incidence } 7 \\
\text { days post-op: } 17 \%\end{array}$ & $\begin{array}{l}\text { Major cardiac } \\
\text { surgery }\end{array}$ & $\begin{array}{c}\text { A } z \text {-score of } \leq-1.96 \\
\text { on at least } 2 \text { different } \\
\text { tests }\end{array}$ & $\begin{array}{l}\text { (i) The short story } \\
\text { module of the } \\
\text { Randt memory } \\
\text { (ii) Grooved } \\
\text { pegboard test } \\
\text { (dominant and } \\
\text { nondominant) } \\
\text { (iii) Digit symbol } \\
\text { subtest } \\
\text { (iv) Trail making } \\
\text { test (part A) } \\
\text { (v) The verbal } \\
\text { fluency test } \\
\text { (vi) Digit span } \\
\text { (forward and } \\
\text { backward) } \\
\text { (vii) Finger } \\
\text { tapping } \\
\text { (viii) Block } \\
\text { subtest }\end{array}$ & $\begin{array}{l}\text { (i) Baseline: } \\
\text { (ii) } 1 \text { day } \\
\text { before } \\
\text { surgery } \\
\text { (iii) Post-op: } \\
\text { (iv) } 7 \text { days }\end{array}$ \\
\hline $\begin{array}{l}\text { Holmgaard } \\
\text { et al., } 2019 \\
{[22]}\end{array}$ & $\begin{array}{l}\text { Secondary analysis } \\
\text { of a randomized } \\
\text { trial } \\
\text { Cerebral oximetry } \\
\text { and POCD }\end{array}$ & $\begin{array}{l}153 \text { (138 male/15 } \\
\text { female) } \\
\geq 55 \text { years } \\
\text { POCD incidence: } \\
29 \% \text { at discharge, } \\
8 \% \text { after } 3 \text { months }\end{array}$ & $\begin{array}{l}\text { Cardiac surgery } \\
\text { (heart valve } \\
\text { surgery, coronary } \\
\text { artery bypass } \\
\text { grafting, or both) }\end{array}$ & $\begin{array}{l}\text { Two out of seven } z \text { - } \\
\text { scores for individual } \\
\text { tests or the composite } \\
z \text {-score }>1.96 \text {. }\end{array}$ & $\begin{array}{l}\text { (i) MMSE ( } \leq 24 \\
\text { excluded) } \\
\text { (ii) Visual verbal } \\
\text { learning test } \\
\text { (iii) Concept } \\
\text { shifting test } \\
\text { (iv) Stroop color } \\
\text { word interference } \\
\text { (SCWI) test } \\
\text { (v) Letter digit } \\
\text { coding (LCD) test }\end{array}$ & $\begin{array}{l}\text { (i) Baseline: } \\
\text { (ii) Before } \\
\text { surgery } \\
\text { (iii) Post-op: } \\
\text { (iv) Day } \\
\text { before } \\
\text { discharge/ } \\
\text { after } 8 \text { days } \\
\text { (v) } 2-4 \\
\text { months }\end{array}$ \\
\hline $\begin{array}{l}\text { Hongyu et al., } \\
2019 \text { [23] }\end{array}$ & $\begin{array}{l}\text { Cohort study } \\
\text { Effect of } \\
\text { penehyclidine } \\
\text { hydrochloride on } \\
\text { POCD }\end{array}$ & $\begin{array}{c}90 \text { (47 male/43 } \\
\text { female) } \\
\text { >60 years } \\
\text { POCD incidence } 7 \\
\text { days post-op: } \\
\text { A } 53.3 \% \\
\text { (penehyclidine } \\
\text { hydrochloride) } \\
\text { B } 26.7 \% \text { (atropine) } \\
\text { C } 13.3 \% \text { (saline) }\end{array}$ & $\begin{array}{l}\text { Thoracoscopic } \\
\text { surgery for lung } \\
\text { cancer }\end{array}$ & $\begin{array}{c}\text { Uneducated }<17 \\
\text { points, primary } \\
\text { education }<20 \text { points, } \\
\text { and }>6 \text { years } \\
\text { education }<24 \text { points. }\end{array}$ & (i) MMSE & $\begin{array}{l}\text { (i) Baseline: } \\
\text { (ii) } 1 \text { day } \\
\text { before } \\
\text { surgery } \\
\text { (iii) Post-op: } \\
\text { (iv) } 1,4 \text {, and } 7 \\
\text { days }\end{array}$ \\
\hline $\begin{array}{l}\text { Kristek et al., } \\
2019 \text { [24] }\end{array}$ & $\begin{array}{c}\text { Prospective RCT } \\
\text { Incidence of POCD } \\
\text { effect } \\
\text { levobupivacaine } \\
\text { versus morphine }\end{array}$ & $\begin{array}{c}70 \text { (4 male/66 } \\
\text { female) } \\
\geq 65 \text { years } \\
\text { POCD incidence } \\
\text { day 1-discharge: } \\
\text { levobupivacaine: } 9 \% \\
\text { Morphine: } 31 \%\end{array}$ & $\begin{array}{l}\text { Femoral fracture } \\
\text { fixation }\end{array}$ & $\begin{array}{l}\text { A decline in the } \\
\text { MMSE score below } \\
17 \text { or a reduction in } \\
\text { the MMSE score for } \\
\geq 3 \text { compared with } \\
\text { the baseline on at } \\
\text { least one } \\
\text { measurement. }\end{array}$ & (i) MMSE & $\begin{array}{l}\text { (i) Baseline: } \\
\text { (ii) Before } \\
\text { surgery } \\
\text { (iii) Post-op: } \\
\text { (iv) Days 1-5 } \\
\text { (v) Day of } \\
\text { discharge }\end{array}$ \\
\hline
\end{tabular}


TABle 1: Continued.

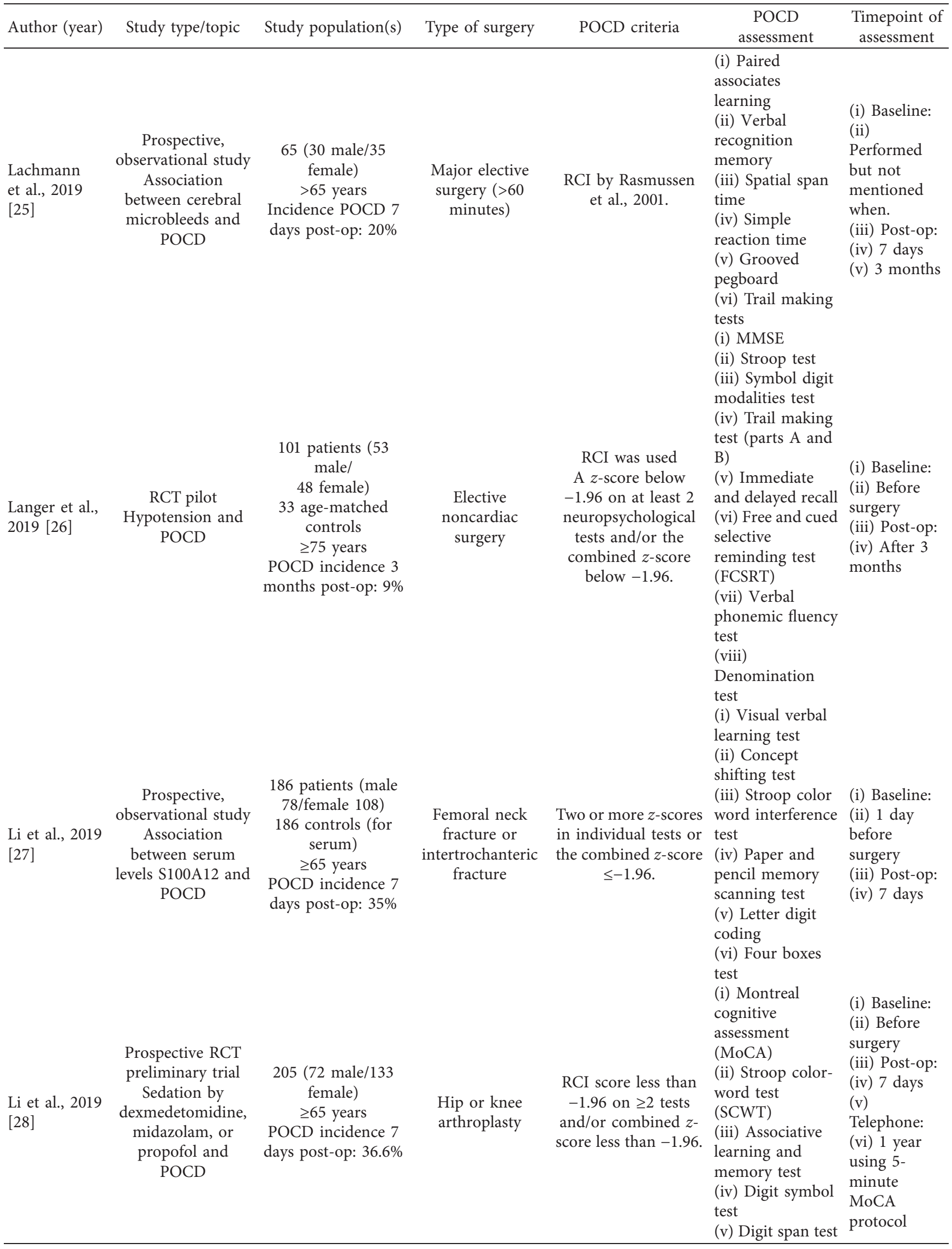


TABle 1: Continued.

\begin{tabular}{|c|c|c|c|c|c|c|}
\hline Author (year) & Study type/topic & Study population(s) & Type of surgery & POCD criteria & $\begin{array}{c}\text { POCD } \\
\text { assessment }\end{array}$ & $\begin{array}{c}\text { Timepoint of } \\
\text { assessment }\end{array}$ \\
\hline $\begin{array}{l}\text { Quan et al., } \\
2019 \text { [29] }\end{array}$ & $\begin{array}{c}\text { RCT } \\
\text { Light vs. deep } \\
\text { anesthesia and } \\
\text { POCD }\end{array}$ & $\begin{array}{c}120 \text { (66 male/54 } \\
\text { female) } \\
\geq 60 \text { years } \\
\text { POCD incidence } 7 \\
\text { days post-op: } \\
\text { Deep: } 19.2 \% \\
\text { Light: } 39.6 \%\end{array}$ & $\begin{array}{l}\text { Abdominal } \\
\text { surgery }\end{array}$ & $\begin{array}{l}\text { Deterioration in } \\
\text { postoperative } \\
\text { performance by } 1 \text { or } \\
\text { more standard } \\
\text { deviations on } 2 \text { or } \\
\text { more tests. }\end{array}$ & $\begin{array}{l}\text { (i) Mental control } \\
\text { (ii) Digit span } \\
\text { (backward and } \\
\text { forward) } \\
\text { (iii) Paired } \\
\text { associate verbal } \\
\text { learning } \\
\text { (iv) Digit symbol } \\
\text { subtest } \\
\text { (v) Visual } \\
\text { retention- } \\
\text { Halstead-Reitan } \\
\text { trail making test } \\
\text { (part A)-grooved } \\
\text { pegboard test }\end{array}$ & $\begin{array}{l}\text { (i) Baseline: } \\
\text { (ii) Before } \\
\text { surgery } \\
\text { (iii) Post-op: } \\
\text { (iv) } 7 \text { days } \\
\text { (v) } 3 \text { months }\end{array}$ \\
\hline $\begin{array}{l}\text { Sánchez et al., } \\
2019 \text { [30] }\end{array}$ & $\begin{array}{l}\text { Longitudinal study } \\
\text { Optimization of } \\
\text { delirium } \\
\text { assessment }\end{array}$ & $\begin{array}{c}1500 \\
\geq 70 \text { years } \\
\text { POCD incidence: }\end{array}$ & $\begin{array}{c}\text { Elective surgery } \\
\text { Thoracic, cardiac, } \\
\text { vascular, proximal } \\
\text { big joints and } \\
\text { spine, } \\
\text { gastrointestinal, } \\
\text { genitourinary, and } \\
\text { general elective } \\
\text { surgery }\end{array}$ & $\begin{array}{l}\text { A test value } \leq 0.5 \\
\text { standard deviations, } \\
\text { normalized for age, } \\
\text { gender, and } \\
\text { education, in one of } \\
\text { these tests. }\end{array}$ & $\begin{array}{l}\text { (i) Montreal } \\
\text { cognitive } \\
\text { assessment } \\
\text { (MoCA) } \\
\text { (ii) Digit span } \\
\text { backwards } \\
\text { (iii) Trail making } \\
\text { test A and B }\end{array}$ & $\begin{array}{l}\text { (i) Baseline: } \\
\text { (ii) } \\
\text { Preadmission } \\
\text { (iii) Before } \\
\text { surgery } \\
\text { (iv) Post-op: } \\
\text { (v) 2, 6, and } \\
12 \text { months }\end{array}$ \\
\hline $\begin{array}{l}\text { Wang et al., } \\
2019[31]\end{array}$ & $\begin{array}{c}\text { Prospective cohort } \\
\text { study } \\
\text { Glucocorticoid } \\
\text { receptor, FKBP51, } \\
\text { and POCD in } \\
\text { elderly }\end{array}$ & $\begin{array}{c}111 \text { (49 male/62 } \\
\text { female) } \\
\geq 65 \text { years } \\
\text { POCD incidence } 7 \\
\text { days post-op: } 28.3 \%\end{array}$ & $\begin{array}{l}\text { Coronary artery } \\
\text { bypass grafting }\end{array}$ & $\begin{array}{c}\text { Reduction in } \\
\text { postoperative test } \\
\text { score compared with } \\
\text { the preoperative test } \\
\text { score } \geq 20 \% \text { in } \geq 2 \\
\text { tests. }\end{array}$ & $\begin{array}{l}\text { (i) MMSE } \\
\text { (ii) Verbal } \\
\text { learning } \\
\text { (iii) Mental } \\
\text { control } \\
\text { (iv) Digit span } \\
\text { (forward and } \\
\text { backward) } \\
\text { (v) Visual } \\
\text { retention } \\
\text { (vi) Grooved } \\
\text { pegboard test } \\
\text { (both hands) } \\
\text { (vii) Digit symbol } \\
\text { test } \\
\text { (viii) Trail making } \\
\text { test (part A) } \\
\text { (i) Stroop color } \\
\text { and word test } \\
\text { (ii) Trail making } \\
\text { test } \\
\text { (iii) Digit symbol } \\
\text { substitution test } \\
\text { (iv) Verbal } \\
\text { learning test } \\
\text { (v) The symbol } \\
\text { digits modalities } \\
\text { test }\end{array}$ & $\begin{array}{l}\text { (i) Baseline: } \\
\text { (ii) } 1 \text { day } \\
\text { before } \\
\text { surgery } \\
\text { (iii) Post-op: } \\
\text { (iv) } 7 \text { days }\end{array}$ \\
\hline $\begin{array}{l}\text { Wang et al., } \\
2019[33]\end{array}$ & $\begin{array}{c}\text { Prospective } \\
\text { observational } \\
\text { cohort study } \\
\text { Smoking history } \\
\text { and risk for POCD }\end{array}$ & $\begin{array}{c}382 \text { (189 male/193 } \\
\text { female) } \\
\geq 60 \text { years } \\
\text { POCD incidence } 5 \\
\text { days post-op: } 50.4 \%\end{array}$ & $\begin{array}{c}\text { Noncardiac } \\
\text { surgery }\end{array}$ & A $z$-score of $\geq 1.96$ & $\begin{array}{l}\text { (i) MMSE } \\
\text { (ii) Concept } \\
\text { shifting test } \\
\text { (iii) Stroop color- } \\
\text { word interference } \\
\text { test }\end{array}$ & $\begin{array}{l}\text { (i) Baseline: } \\
\text { (ii) } 1 \text { day } \\
\text { before } \\
\text { surgery } \\
\text { (iii) Post-op: } \\
\text { (iv) } 5 \text { and } 7 \\
\text { days }\end{array}$ \\
\hline
\end{tabular}


TABLE 1: Continued.

\begin{tabular}{|c|c|c|c|c|c|c|}
\hline Author (year) & Study type/topic & Study population(s) & Type of surgery & POCD criteria & $\begin{array}{c}\text { POCD } \\
\text { assessment }\end{array}$ & $\begin{array}{c}\text { Timepoint of } \\
\text { assessment }\end{array}$ \\
\hline $\begin{array}{l}\text { Wang et al., } \\
2019 \text { [34] }\end{array}$ & $\begin{array}{c}\text { RCT } \\
\text { Ropivacaine } \\
\text { intercostal nerve } \\
\text { block and POCD }\end{array}$ & $\begin{array}{c}100 \text { (50 male/50 } \\
\text { female) } \\
\text { Age: } 43-46 \text { years } \\
\text { POCD incidence: } \\
\text { With block: } 21 \% \\
\text { Without block: } 39 \%\end{array}$ & $\begin{array}{l}\text { Thoracotomy for } \\
\text { esophageal cancer }\end{array}$ & $\begin{array}{l}\text { Score less than or } \\
\text { equal to } 25 \text { points, or } \\
\text { if more than } 2 \text { points } \\
\text { reduction on the } \\
\text { MMSE score after } \\
\text { surgery compared } \\
\text { with the score before } \\
\text { surgery. }\end{array}$ & (i) MMSE & $\begin{array}{l}\text { (i) Baseline: } \\
\text { (ii) } 1 \text { hour } \\
\text { before } \\
\text { surgery } \\
\text { (iii) Post-op: } \\
\text { (iv) } 2,12 \text {, and } \\
24 \text { hours }\end{array}$ \\
\hline $\begin{array}{l}\text { Zhang et al., } \\
2019 \text { [35] }\end{array}$ & $\begin{array}{c}\text { Cohort study } \\
\text { Effects of fentanyl } \\
\text { versus sufentanil on } \\
\text { POCD }\end{array}$ & $\begin{array}{c}96 \text { (47 male/49 } \\
\text { female) } \\
\geq 65 \text { years } \\
\text { POCD incidence } 7 \\
\text { days post-op: } \\
\text { Fentanyl: } 11.9 \% \\
\text { Sufentanil: } 6.2 \%\end{array}$ & Open surgery & $\begin{array}{l}\text { A drop of } 1 \text { standard } \\
\text { deviation from } \\
\text { baseline on two or } \\
\text { more items. }\end{array}$ & (i) $\mathrm{MoCA}$ & $\begin{array}{l}\text { (i) Baseline: } \\
\text { (ii) } 1 \text { day } \\
\text { before } \\
\text { surgery } \\
\text { (iii) Post-op: } \\
\text { (iv) } 1,7 \text { days }\end{array}$ \\
\hline $\begin{array}{l}\text { Zhang et al., } \\
2019 \text { [36] }\end{array}$ & $\begin{array}{l}\text { Cohort study } \\
\text { Risk factors for } \\
\text { POCD }\end{array}$ & $\begin{array}{c}77 \text { (42 male/35 } \\
\text { female) } \\
\geq 65 \text { years } \\
\text { POCD } 7 \text { days post- } \\
\text { op: } 24.7 \%\end{array}$ & Colorectal surgery & $\begin{array}{l}z \text {-score was greater } \\
\text { than } 1.96 \text { or the } \\
\text { combined } z \text { - score } \\
\text { was } \geq 1.96 \text {. }\end{array}$ & $\begin{array}{l}\text { (i) MMSE } \\
\text { (ii) Visual verbal } \\
\text { learning test } \\
\text { (iii) Digit span } \\
\text { test } \\
\text { (iv) Digit symbol } \\
\text { substitution test }\end{array}$ & $\begin{array}{l}\text { (i) Baseline: } \\
\text { (ii) } 1 \text { day } \\
\text { before } \\
\text { surgery } \\
\text { (iii) Post-op: } \\
\text { (iv) } 7 \text { days }\end{array}$ \\
\hline
\end{tabular}

backward test $n=14$ ), which measures short-term memory, the trail making test part A $(n=13)$, which tests processing speed and mental flexibility by connecting numbered dots in sequence, and the digit symbol substitution test $(n=11)$, which measures visuoperceptual functions and motor speed.

Not only the used tests and timing of testing differed considerably between studies, but also the criteria to define a "positive" test result. While some authors use a deterioration compared to baseline testing in terms of the absolute test score (e.g., >2-point deterioration in the MMSE), other authors define a positive test result in terms of the deterioration of $\geq 1$ standard deviation from the baseline measurement. Other authors use $z$-scores, in which the difference in the observed change from baseline between surgical patients and control patients is scaled by the standard deviation of control patients [38]. The "reliable change index" (RCI) - a measure of change from baseline in units of the standard error of the change [39] — has occasionally been used in addition to, or instead of, $z$-scores.

\section{Discussion}

Symptoms of cognitive dysfunction are estimated to occur postoperatively in about $12 \%$ of patients without apparent preoperative cognitive dysfunction undergoing noncardiac surgery [4], and the incidence may be as high as $50-70 \%$ after cardiac surgery [40]. While these symptoms are often transient, cognitive impairment has been observed in up to $10 \%$ of elderly patients at 3 months after surgery [4], and it has been estimated that about one-half of elderly patients with POCD suffer permanent dysfunction [41]. POCD is thus evidently a major threat after an operation in particular to elderly patients, and every effort is needed to prevent, diagnose, and treat POCD in this vulnerable patient population.

Unfortunately, the pathophysiology and risk factors are still poorly understood, and evidence-based treatment options are scarce. It is thus not surprising that POCD is a target of an increasing amount of research papers in anesthesiologic, surgical, and neurologic literature. Using the search term "postoperative cognitive dysfunction" in PubMed yields almost 4,000 results at the time of the writing of this manuscript, with a steady increase over the years: while there were 50 publications in the year 2000, this has increased to 168 in 2010 and to 426 in 2019. The literature, however, has in the past been very heterogenous in how POCD was defined, and this is a major threat to clinical research in this area: when the condition being studied is not well characterized and well defined, study results may not be readily comparable and applicable to clinical practice. Moreover, there are several types of cognitive impairments that can be observed after an operation, including delirium, POCD, and dementia. These terms are sometimes used interchangeably or with a broad overlap in the literature, and a clear distinction is often lacking. In 2010, Rudolph and colleagues have demonstrated a substantial inconsistency in diagnostic criteria, neurocognitive tests, and timing of the assessment of POCD across studies in cardiac surgery [5]. Since then, multiple authors have emphasized the need for uniform terminology and diagnostic criteria in POCD research. However, while it is clear that a uniform approach to defining POCD was previously lacking, it is unclear whether this issue has been successfully improved or even resolved in recent years.

In this focused systematic review, we therefore aimed to study how POCD is defined and measured in the recent 
TABle 2: Neurocognitive tests used in the included studies to define POCD.

\begin{tabular}{|c|c|}
\hline Neurocognitive test & Number of studies, in which the test was used \\
\hline Associative learning and memory test & 1 \\
\hline Auditory verbal learning test & 2 \\
\hline Block subtest & 1 \\
\hline Clock drawing test & 2 \\
\hline Concept shifting test & 4 \\
\hline Controlled oral word association test & 1 \\
\hline Denomination test & 1 \\
\hline Digit symbol substitution test & 11 \\
\hline Digit span test & 13 \\
\hline Forward & $\begin{array}{l}13 \\
14\end{array}$ \\
\hline Backward & 14 \\
\hline Finger tapping test & 1 \\
\hline Four boxes test & 1 \\
\hline Free and cued selective reminding test & 1 \\
\hline Grooved pegboard test & 7 \\
\hline Hopkins verbal learning test & 1 \\
\hline Immediate recall and delayed recall & 2 \\
\hline Letter digit coding test & 2 \\
\hline Letter digit substitution test & 1 \\
\hline Logical memory test & 1 \\
\hline Mental control & 4 \\
\hline MMSE & 14 \\
\hline $\mathrm{MoCA}$ & 6 \\
\hline Paired association verbal learning test & 5 \\
\hline Paper pencil memory scanning test & 1 \\
\hline Stroop color interference test & 7 \\
\hline Short story module of the Randt memory & 1 \\
\hline Simple reaction time & 1 \\
\hline Spatial span time & 1 \\
\hline Symbol digits modalities test & 1 \\
\hline Trail making test & 13 \\
\hline Part A & $\begin{array}{c}13 \\
6\end{array}$ \\
\hline Part B & \\
\hline Verbal fluency test & 3 \\
\hline Verbal learning test & 4 \\
\hline Visuospatial memory test (brief) & 1 \\
\hline Visuospatial skill test & 1 \\
\hline Visual retention & 4 \\
\hline Visual verbal learning test (here: with capital letters) & 4 \\
\hline Word list generation test & 1 \\
\hline Word recognition memory test & 1 \\
\hline
\end{tabular}

literature. The results suggest that even in the most recent POCD literature, a consistent approach towards defining and measuring POCD is still lacking. As previously noted by other authors [4, 42, 43], uniform diagnostic criteria are needed, and this is clearly reinforced by our data.

While our systematic review identified the lack of a consistent approach towards defining POCD, the data, however, provide more than a mere description of heterogeneity across recent studies. We observed some patterns that may serve as a common denominator in the process of reaching standardized diagnostic criteria: with respect to timing, a follow-up measurement at 7 days postoperatively seems to be broadly accepted. With respect to the tests being used, the MMSE, digit span test, trail making test part A, and the digit symbol substitution test were used most commonly, suggesting a broad consensus that these tests are particularly useful for the diagnosis of postoperative neurocognitive disorders. However, it is still unclear which test result would be considered "positive" for POCD. Moreover, it should be noted that these tests have not been developed for POCD testing and have also not been rigorously validated for this purpose. For example, the MMSE has been shown to be less sensitive than the MoCA for a variety of neurocognitive disorders [44], and this could also be true for POCD. This implies that more research not only on the pathophysiology, prevention, and treatment of POCD is required, but also on the diagnostic accuracy [45] of the tests themselves that are commonly used to diagnose POCD.

Our data suggest that strong efforts are necessary to define precise and applicable diagnostic criteria for POCD, involving key-opinion leaders and researchers in the field. This would, in our opinion, be a strong and necessary step towards a precise characterization of the disease, its underlying pathophysiology, risk factors, and treatment 
options. Notably, however, perhaps the term POCD itself is too broad and vague and actually represents several distinct neuropathologic conditions, rather than one underlying common pathophysiology. If so, this may have important implications for the identification of risk factors as well as prevention and treatment strategies and may explain the heterogeneity in findings across the literature. In this context, Evered et al. have previously suggested that the overarching term POCD should be changed to "delayed neurocognitive recovery" for symptoms expected to have been resolved before 30 days and "postoperative mild neurocognitive disorder" or "postoperative major neurocognitive disorder" for an expected recovery between 30 days and 12 months, depending on the severity of symptoms [6]. Therefore, future efforts should perhaps not so much focus on defining diagnostic criteria for the overarching concept of "POCD" itself, but rather more specifically, for distinct postoperative neurocognitive disorders comprised within this umbrella term. Moreover, it is also well possible that some cognitive domains may be more vulnerable after surgery and anesthesia than others, or that a cognitive decline in some domains may affect patients more severely than a decline in other domains. We believe that identification of those domains that are particularly relevant in the context of POCD is an important topic for future research because broad testing for a decline in all cognitive domains may neither be necessary nor ideal. Focusing on those domains which are most vulnerable and/or most severely affect the patients' wellbeing would reduce the patients' burden to undergo a whole battery of neurocognitive tests, facilitate clinical testing, and allow for a more efficient allocation of clinical and research resources.

The results of this systematic review must be viewed in the context of its limitations. First, this was a focused review, deliberately limited to the literature published in the last two years, and it thus does not claim to be an exhaustive review of all published literature on the topic. However, we specifically attempted to assess how POCD is currently diagnosed, and older literature would merely have been of historic interest. We only searched one database, however, one with comprehensive coverage of reputable medical literature. It was neither our intention to identify gray literature on the topic, nor to identify literature published in predatory or otherwise untrustworthy literature. We are also aware that the mere fact that a majority of studies used a certain definition to diagnose POCD does not necessarily mean that this is the optimal way to diagnose POCD. It does, however, suggest that there is a rather broad consensus among researchers that certain tests at certain time points may play a useful role and can serve as a starting point towards a more uniform definition and assessment of POCD in clinical practice and in research settings.

In conclusion, this systematic review identified the lack of a consistent approach towards defining POCD. Commonalities were identified which may serve as a common denominator for deriving consensus based diagnostic guidelines for POCD. However, more research is necessary to characterize the diagnostic accuracy of the tests used to identify a postoperative cognitive decline and on what would constitute a "positive" test result. Finally, future efforts should perhaps not so much focus on finding a uniform definition of POCD itself, but rather on characterizing and defining distinct postoperative neurocognitive disorders comprised within this overarching term.

\section{Data Availability}

All data are extracted from the published literature, which are available from the respective publisher upon request.

\section{Conflicts of Interest}

Kim van Sinderen, Lothar A. Schwarte, and Patrick Schober report no conflicts of interest regarding the publication of this paper.

\section{Authors' Contributions}

Kim van Sinderen helped in designing the study, conducting the study, analyzing the data, and writing the manuscript. Lothar A. Schwarte helped in conducting the study, analyzing the data, and writing the manuscript. Patrick Schober helped in designing the study, analyzing the data, and writing the manuscript.

\section{References}

[1] A.-M. Sauër, C. Kalkman, and D. van Dijk, "Postoperative cognitive decline," Journal of Anesthesia, vol. 23, no. 2, pp. 256-259, 2009.

[2] L. Evered, B. Silbert, D. A. Scott, D. Ames, P. Maruff, and K. Blennow, "Cerebrospinal fluid biomarker for alzheimer disease predicts postoperative cognitive dysfunction," Anesthesiology, vol. 124, no. 2, pp. 353-361, 2016.

[3] L. A. Evered and B. S. Silbert, "Postoperative cognitive dysfunction and noncardiac surgery," Anesthesia \& Analgesia, vol. 127, no. 2, pp. 496-505, 2018.

[4] M. J. Needham, C. E. Webb, and D. C. Bryden, "Postoperative cognitive dysfunction and dementia: what we need to know and do," British Journal of Anaesthesia, vol. 119, no. suppl_1, pp. i115-i125, 2017.

[5] J. L. Rudolph, K. A. Schreiber, D. J. Culley et al., "Measurement of post-operative cognitive dysfunction after cardiac surgery: a systematic review," Acta Anaesthesiologica Scandinavica, vol. 54, no. 6, pp. 663-677, 2010.

[6] L. Evered, B. Silbert, D. S. Knopman et al., "Recommendations for the nomenclature of cognitive change associated with anaesthesia and surgery-2018," Anesthesia \& Analgesia, vol. 127, no. 5, pp. 1189-1195, 2018.

[7] A. J. Claes, S. de Backer, P. Van de Heyning, A. Gilles, V. Van Rompaey, and G. Mertens, "Postoperative cognitive dysfunction after cochlear implantation," European Archives of Oto-Rhino-Laryngology, vol. 275, no. 6, pp. 1419-1427, 2018.

[8] X. Duan, T. Zhu, C. Chen et al., "Serum glial cell line-derived neurotrophic factor levels and postoperative cognitive dysfunction after surgery for rheumatic heart disease," The Journal of Thoracic and Cardiovascular Surgery, vol. 155, no. 3, pp. 958-965, 2018.

[9] G.-L. Gong, B. Liu, J.-X. Wu, J.-Y. Li, B.-Q. Shu, and Z.-J. You, "Postoperative cognitive dysfunction induced by different 
surgical methods and its risk factors," The American Surgeon, vol. 84, no. 9, pp. 1531-1537, 2018.

[10] K. Hayashi, H. Oshima, M. Shimizu et al., "Preoperative 6minute walk distance is associated with postoperative cognitive dysfunction," The Annals of Thoracic Surgery, vol. 106, no. 2, pp. 505-512, 2018.

[11] R. Hou, H. Wang, L. Chen, Y. Qiu, and S. Li, "POCD in patients receiving total knee replacement under deep vs light anesthesia: a randomized controlled trial," Brain and Behavior, vol. 8, no. 2, Article ID e00910, 2018.

[12] Y. Konishi, L. A. Evered, D. A. Scott, and B. S. Silbert, "Postoperative cognitive dysfunction after sevoflurane or propofol general anaesthesia in combination with spinal anaesthesia for hip arthroplasty," Anaesthesia and Intensive Care, vol. 46, no. 6, pp. 596-600, 2018.

[13] B. Kumpaitiene, M. Svagzdiene, I. Drigotiene et al., "Correlation among decreased regional cerebral oxygen saturation, blood levels of brain injury biomarkers, and cognitive disorder," Journal of International Medical Research, vol. 46, no. 9, pp. 3621-3629, 2018.

[14] C. Sahan, Z. Sungur, E Camci et al., "Effects of cerebral oxygen changes during coronary bypass surgery on postoperative cognitive dysfunction in elderly patients: a pilot study," Brazilian Journal of Anesthesiology, vol. 68, no. 2, pp. 142-148, 2018.

[15] N. Zhang, M. Liang, D.-d. Zhang et al., "Effect of goal-directed fluid therapy on early cognitive function in elderly patients with spinal stenosis: a case-control study," International Journal of Surgery, vol. 54, no. Pt A, pp. 201-205, 2018.

[16] Y. Zhang, G.-J. Shan, Y.-X. Zhang et al., "Propofol compared with sevoflurane general anaesthesia is associated with decreased delayed neurocognitive recovery in older adults," British Journal of Anaesthesia, vol. 121, no. 3, pp. 595-604, 2018.

[17] Y. Zhang, G.-J. Shan, Y.-X. Zhang et al., "Preoperative vitamin $\mathrm{D}$ deficiency increases the risk of postoperative cognitive dysfunction: a predefined exploratory sub-analysis," Acta Anaesthesiologica Scandinavica, vol. 62, no. 7, pp. 924-935, 2018.

[18] X. Q. Cheng, B. Mei, Y. M. Zuo et al., "A multicentre randomised controlled trial of the effect of intra-operative dexmedetomidine on cognitive decline after surgery," $A n$ aesthesia, vol. 74, no. 6, pp. 741-750, 2019.

[19] L. A. Daiello, A. M. Racine, R. Yun Gou et al., "Postoperative delirium and postoperative cognitive dysfunction," Anesthesiology, vol. 131, no. 3, pp. 477-491, 2019.

[20] B. Gao, B. Zhu, and C. Wu, "Preoperative serum 25hydroxyvitamin D level, a risk factor for postoperative cognitive dysfunction in elderly subjects undergoing total joint arthroplasty," The American Journal of the Medical Sciences, vol. 357, no. 1, pp. 37-42, 2019.

[21] Y. Han, L. Han, M.-M. Dong et al., "Preoperative salivary cortisol AM/PM ratio predicts early postoperative cognitive dysfunction after noncardiac surgery in elderly patients," Anesthesia \& Analgesia, vol. 128, no. 2, pp. 349-357, 2019.

[22] F. Holmgaard, A. G. Vedel, L. S. Rasmussen, O. B. Paulson, J. C. Nilsson, and H. B. Ravn, "The association between postoperative cognitive dysfunction and cerebral oximetry during cardiac surgery: a secondary analysis of a randomised trial," British Journal of Anaesthesia, vol. 123, no. 2, pp. 196-205, 2019.

[23] X. Hongyu, W. Qingting, S. Xiaoling, Z. Liwu, Y. Ailing, and L. Xin, "Penehyclidine hydrochloride on postoperatively cognitive function," Medical Hypotheses, vol. 129, Article ID 109246, 2019.

[24] G. Kristek, I. Radoš, D. Kristek et al., "Influence of postoperative analgesia on systemic inflammatory response and postoperative cognitive dysfunction after femoral fractures surgery: a randomized controlled trial," Regional Anesthesia \& Pain Medicine, vol. 44, no. 1, pp. 59-68, 2019.

[25] G. Lachmann, I. Kant, F. Lammers et al., "Cerebral microbleeds are not associated with postoperative delirium and postoperative cognitive dysfunction in older individuals," PLoS One, vol. 14, no. 6, Article ID e0218411, 2019.

[26] T. Langer, A. Santini, F. Zadek et al., "Intraoperative hypotension is not associated with postoperative cognitive dysfunction in elderly patients undergoing general anesthesia for surgery: results of a randomized controlled pilot trial," Journal of Clinical Anesthesia, vol. 52, pp. 111-118, 2019.

[27] Q. H. Li, L. Yu, Z. W Yu et al., "Relation of postoperative serum S100A12 levels to delirium and cognitive dysfunction occurring after hip fracture surgery in elderly patients," Brain and Behavior, vol. 9, no. 1, Article ID e01176, 2019.

[28] W.-X. Li, R.-Y. Luo, C. Chen et al., "Effects of propofol, dexmedetomidine, and midazolam on postoperative cognitive dysfunction in elderly patients," Chinese Medical Journal, vol. 132, no. 4, pp. 437-445, 2019.

[29] C. Quan, J. Chen, Y Luo et al., "BIS-guided deep anesthesia decreases short-term postoperative cognitive dysfunction and peripheral inflammation in elderly patients undergoing abdominal surgery," Brain and Behavior, vol. 9, no. 4, Article ID e01238, 2019.

[30] A. Sanchez, C. Thomas, F. Deeken et al., "Patient safety, costeffectiveness, and quality of life: reduction of delirium risk and postoperative cognitive dysfunction after elective procedures in older adults-study protocol for a stepped-wedge cluster randomized trial (PAWEL Study)," Trials, vol. 20, no. 1, p. 71, 2019.

[31] L. W. Wang, M. J. Zhu, Y Li et al., "FKBP51 is associated with early postoperative cognitive dysfunction in elderly patients undergoing hip fracture surgery," Medicine, vol. 98, no. 5, Article ID e14037, 2019.

[32] M. Wang, P. Su, Y Liu et al., "Abnormal expression of circRNA_089763 in the plasma exosomes of patients with postoperative cognitive dysfunction after coronary artery bypass grafting," Molecular Medicine Reports, vol. 20, no. 3, pp. 2549-2562, 2019.

[33] R. Wang, G. Wang, Y. Liu, and M. Zhang, "Preoperative smoking history is associated with decreased risk of early postoperative cognitive dysfunction in patients of advanced age after noncardiac surgery: a prospective observational cohort study," Journal of International Medical Research, vol. 47, no. 2, pp. 689-701, 2019.

[34] Y. Wang, J. Cheng, L. Yang, J. Wang, H. Liu, and Z. Lv, "Ropivacaine for intercostal nerve block improves early postoperative cognitive dysfunction in patients following thoracotomy for esophageal cancer," Medical Science Monitor, vol. 25, pp. 460-465, 2019.

[35] J. Zhang, L. Chen, Y. Sun, and W. He, "Comparative effects of fentanyl versus sufentanil on cerebral oxygen saturation and postoperative cognitive function in elderly patients undergoing open surgery," Aging Clinical and Experimental Research, vol. 31, no. 12, pp. 1791-1800, 2019.

[36] Y. Zhang, H.-G. Bao, Y.-L Lv et al., "Risk factors for early postoperative cognitive dysfunction after colorectal surgery," BMC Anesthesiology, vol. 19, no. 1, p. 6, 2019. 
[37] P. S. Sachdev, D. Blacker, D. G. Blazer et al., "Classifying neurocognitive disorders: the DSM-5 approach," Nature Reviews Neurology, vol. 10, no. 11, pp. 634-642, 2014.

[38] L. S. Rasmussen, K. Larsen, P. Houx et al., "The assessment of postoperative cognitive function," Acta Anaesthesiologica Scandinavica, vol. 45, no. 3, pp. 275-289, 2001.

[39] N. S. Jacobson and P. Truax, "Clinical significance: a statistical approach to defining meaningful change in psychotherapy research," Journal of Consulting and Clinical Psychology, vol. 59, no. 1, pp. 12-19, 1991.

[40] M. F. Newman, J. P. Mathew, H. P. Grocott et al., "Central nervous system injury associated with cardiac surgery," The Lancet, vol. 368, no. 9536, pp. 694-703, 2006.

[41] N. Kotekar, A. Shenkar, and R. Nagaraj, "Postoperative cognitive dysfunction-current preventive strategies," Clinical Interventions in Aging, vol. 13, pp. 2267-2273, 2018.

[42] E. A. Çizmeci and A. J. C. Slooter, "Defining perioperative neurocognitive disorders: still more to clarify," British Journal of Anaesthesia, vol. 123, no. 3, p. e468, 2019.

[43] L. Evered, B. Silbert, D. S. Knopman et al., "Recommendations for the nomenclature of cognitive change associated with anaesthesia and surgery-2018," British Journal of Anaesthesia, vol. 121, no. 5, pp. 1005-1012, 2018.

[44] G. S. A. Siqueira, P. d. M. S. Hagemann, D. d. S. Coelho, F. H. D. Santos, and P. H. F. Bertolucci, "Can MoCA and MMSE be interchangeable cognitive screening tools? A systematic review," The Gerontologist, vol. 59, no. 6, pp. e743-e763, 2019.

[45] T. R. Vetter, P. Schober, and E. J. Mascha, "Diagnostic testing and decision-making," Anesthesia \& Analgesia, vol. 127, no. 4, pp. 1085-1091, 2018. 